\title{
Surface Science in an MOCVD Environment: Arsenic on Vicinal Ge(100)
}

W.E. McMahon and J.M. Olson

National Renewable Energy Laboratory

Presented at the National Center for

Photovoltaics Program Review Meeting Denver, Colorado

September 8-11, 1998

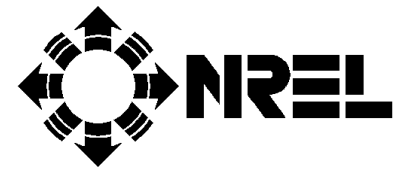

National Renewable Energy Laboratory 1617 Cole Boulevard Golden, Colorado 80401-3393

A national laboratory of the U.S. Department of Energy Managed by Midwest Research Institute for the U.S. Department of Energy under contract No. DE-AC36-83CH10093

Work performed under task number PV904401

November 1998 


\begin{abstract}
NOTICE
This report was prepared as an account of work sponsored by an agency of the United States government. Neither the United States government nor any agency thereof, nor any of their employees, makes any warranty, express or implied, or assumes any legal liability or responsibility for the accuracy, completeness, or usefulness of any information, apparatus, product, or process disclosed, or represents that its use would not infringe privately owned rights. Reference herein to any specific commercial product, process, or service by trade name, trademark, manufacturer, or otherwise does not necessarily constitute or imply its endorsement, recommendation, or favoring by the United States government or any agency thereof. The views and opinions of authors expressed herein do not necessarily state or reflect those of the United States government or any agency thereof.
\end{abstract}

Available to DOE and DOE contractors from:

Office of Scientific and Technical Information (OSTI)

P.O. Box 62

Oak Ridge, TN 37831

Prices available by calling 423-576-8401

Available to the public from:

National Technical Information Service (NTIS)

U.S. Department of Commerce

5285 Port Royal Road

Springfield, VA 22161

703-605-6000 or 800-553-6847

or

DOE Information Bridge

http://www.doe.gov/bridge/home.html 


\title{
Surface Science in an MOCVD Environment: Arsenic on Vicinal Ge(100)
}

\author{
W.E. McMahon and J.M. Olson \\ National Renewable Energy Laboratory, 1617 Cole Blvd., Golden, CO 80401
}

\begin{abstract}
Scanning tunneling microscope (STM) images of arsine-exposed vicinal Ge(100) surfaces show that most $\mathrm{As} / \mathrm{Ge}$ steps are reconstructed, and that a variety of different step structures exist. The entire family of reconstructed $\mathrm{As} / \mathrm{Ge}$ steps can be divided into two types, which we have chosen to call "single-row" steps and "double-row" steps. In this paper we propose a model for a double-row step created by annealing a vicinal $\mathrm{Ge}(100)$ substrate under an arsine flux in a metal-organic chemical vapor deposition (MOCVD) chamber.
\end{abstract}

\section{INTRODUCTION}

Because GaAs and Ge are lattice-matched and have nearly the same coefficients of thermal expansion, GaAs/Ge would seem to be an ideal choice for heteroepitaxy. In particular, vicinal $\mathrm{Ge}(100)$ should provide a good template for GaAs growth. However, even after 30 years of study (1) there is still no fundamental understanding of the nucleation of GaAs on Ge. Instead, peculiar dependencies upon growth chamber design and substrate processing history are observed (2-4).

The most likely source of confusion is that very little is known about the atomic structure of the Ge surface immediately prior to GaAs growth. In most cases, the Ge substrate is either intentionally or unintentionally exposed to As prior to GaAs nucleation. It is known that exposure to As can significantly modify the step structure of vicinal Ge (5), suggesting that the atomic structure of As/Ge (arsenic-exposed $\mathrm{Ge}$ ) steps is different from Ge steps. Nonetheless, an atomic resolution study of $\mathrm{As} / \mathrm{Ge}$ steps has never been performed.

For this reason, we have conducted a very extensive survey of As/Ge steps at atomic resolution with a scanning tunneling microscope (STM). Our data clearly show that $\mathrm{As} / \mathrm{Ge}$ steps reconstruct, and that phase transitions between different As/Ge step reconstructions occur. Although we have observed differences in step structure as a function of As source (arsine vs. As 4 , for example), in this paper we will limit our discussion to arsine-exposed Ge.

\section{EXPERIMENTAL DETAILS}

We have examined arsine-exposed $\mathrm{Ge}(100)$ miscut $2^{\circ}$ and $6^{\circ}$ toward (111) and $6^{\circ}$ toward (110). These samples were annealed in a metal-organic chemical vapor deposition (MOCVD) chamber under 1.2 torr $\mathrm{AsH}_{3}$ diluted in 70.8 torr of $\mathrm{H}_{2}$ carrier gas flowing at $6 \mathrm{~L} / \mathrm{min}$. 
After preparation in the MOCVD chamber, samples were quenched to room temperature and transferred under vacuum to an ultra-high vacuum (UHV) analysis chamber for study with low-energy electron diffraction (LEED), Auger electron spectroscopy (AES), and STM. All of the surfaces we have studied are as-quenched, with no further preparation after removal from the growth chamber.

Though we do not show any LEED images here, it serves as a quick and easy method of determining the phase of a surface. With LEED, one can determine (to varying degrees) the sample roughness, ratio of $(1 \times 2)$ to $(2 \times 1)$ terraces, degree and direction of facetting, and the spacing of any ordered steps.

AES is used to monitor the elemental composition of the surfaces. Using AES, we checked each sample for surface contamination (carbon, oxygen, or indium, for example). In addition, we are able to record the As and Ge peaks, which in principle could be used to determine the As coverages of our As/Ge surfaces. In practice, we have found that the As/Ge Auger peak intensity ratio is nominally $1 / 15$ for most of our As/Ge surfaces (at an incident-beam voltage of $5.0 \mathrm{keV}$ ). Based on published work (6) and the fact that our As/Ge surfaces are chemically quite passive, we have assumed that these surfaces are As-terminated, with an unknown amount of As diffusion into the Ge substrate.

\section{DEFINITIONS}

To accurately describe a step, at least four parameters must be specified: (1) Step height in layers. 1 layer $=1$ Ge monolayer $=1.4145 \AA=a_{0} / 4$, where $a_{0}$ is the lattice constant of Ge. (2) Step type. For type 'A' ('B') steps, the dimer rows on the upper As/Ge terrace run parallel (perpendicular) to the step edge. (3) Step reconstruction. In the course of our work, we have found that the entire family of As/Ge step reconstructions can be divided into two types. We have chosen to call them "singlerow" (SR) reconstructions and "double-row" (DR) reconstructions. In this paper, we will limit our discussion to DR reconstructions. (4) Surface composition. Steps on clean (As-free) Ge are called "Ge steps," whereas steps on arsenic-exposed Ge are called "As/Ge steps."

For clarity, step height and type can be abbreviated. A "2-layer type 'B' " step is called a "2B" step, for example.

\section{RESULTS AND DISCUSSION}

We begin our discussion with the 2B DR As/Ge step shown in Fig. 1. We have chosen this particular step because it is a particularly clear and simple example.

Fig. 1a shows top and side views of our STM data. The terraces consist of dimer rows, as expected. Each dimer site is labeled with a 'D.' 'S1,' 'S2,' and 'S3' denote 


\section{Side View}
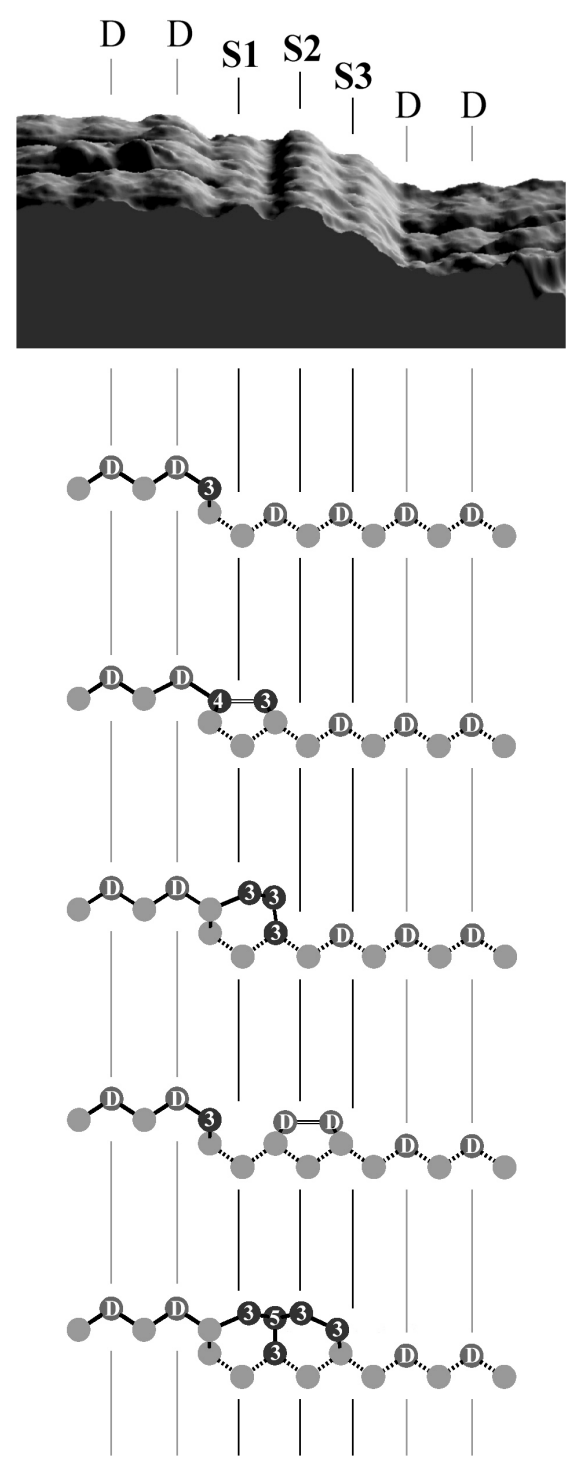

Top View

D D S1 S2 S3 D D

A

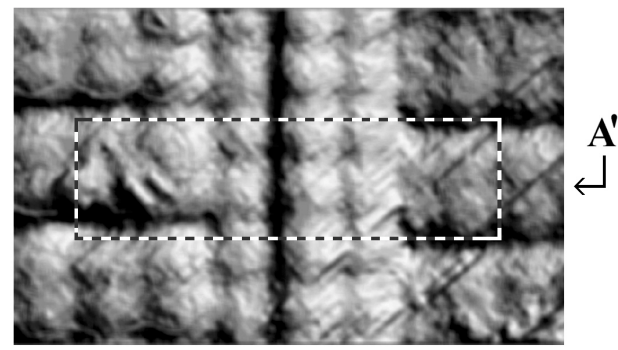

(b)

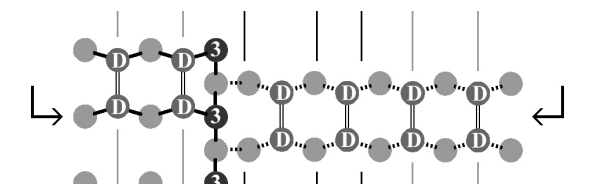

(c)

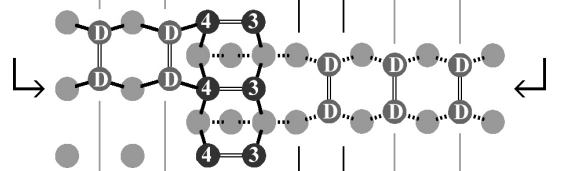

(d)

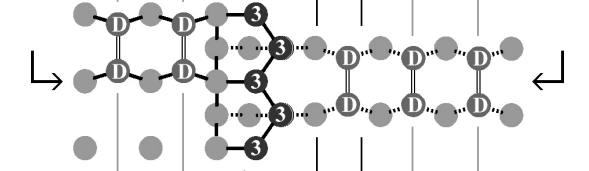

(e)

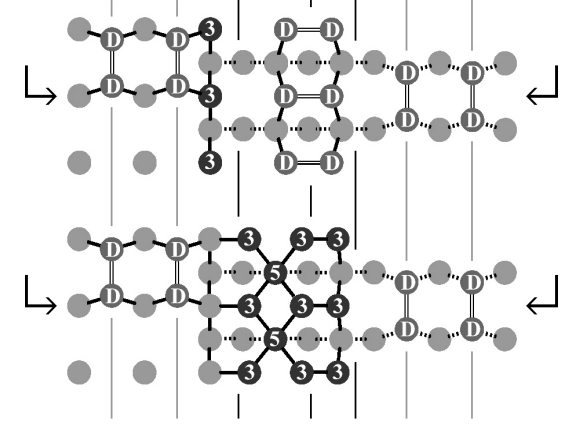

FIGURE 1. (a) A high-resolution image of a $2 \mathrm{~B} \mathrm{DR}$ As/Ge step. To create this step, a Ge(100) $2^{\circ}$ (111) surface was annealed in an MOCVD chamber at $640^{\circ} \mathrm{C}$ for 25 min under 1.2 torr $\mathrm{AsH}_{3}$ partial pressure. These images have been artificially illuminated from the upper right to bring out the atomicresolution details. $\mathrm{V}_{\text {sample }}=-3.0 \mathrm{~V}$ and $\mathrm{I}_{\mathrm{tun}}=1.0 \mathrm{nA}$. Three step sites are labeled 'S1,' 'S2,' and 'S3'. Dimer sites are labeled 'D.'

Models (b) - (f) are aligned below the STM images for ease of comparison. A box in the STM top view defines the area included in each model. The arrows in each top view show the position of line A$\mathbf{A}^{\prime}$. See text for a discussion of these models. 
step sites imaged by the STM tip as it crossed the step edge. This step topology has been observed with different STM tips on many different samples, ruling out the possibility that this image contains double-tip generated "ghost" sites.

We have chosen to call this a "double-row" reconstruction based on the double row of atoms formed by sites 'S1' and 'S2.' As mentioned earlier, we have also observed As/Ge steps with only a single row of 'S1' sites. SR steps will not be discussed in this paper.

Figs. 1b-f are ball-and-stick models presented for comparison. These will be referred to as models $1 \mathrm{~b}-1 \mathrm{f}$. A ' $\mathrm{D}$ ' denotes a dimer atom, whereas numbered sites are labeled with the number of nearest neighbors. Bulk-like or nearly bulk-like sites are unlabeled. The chemical identity of individual sites has been left unlabeled. The lines in our diagrams simply connect nearest-neighbor sites and do not necessarily correspond to chemical bonds.

To first order, we are only interested in the geometry of the various structures, so we considered existing models for $\mathrm{Ge}, \mathrm{Si}$, and $\mathrm{As} / \mathrm{Si} 2 \mathrm{~B}$ steps. Model $\mathrm{1b}$ is a bulk-like $2 \mathrm{~B}$ step (7), model $1 \mathrm{c}$ is a rebonded $2 \mathrm{~B}$ step (8), and model $1 \mathrm{~d}$ is a pi-bonded $2 \mathrm{~B}$ step (9). None of these three models explains site ' $\mathrm{S} 3$,' and model $1 \mathrm{~b}$ cannot even explain site 'S2.'

Although these differences are most easily seen in the side views, they are also visible in the top views. For comparison, the line $\mathbf{A}-\mathbf{A}^{\prime}$ has been indicated with arrows in each top view. In our STM image, all three step sites lie along this line. In contrast, models $1 \mathrm{~b}-\mathrm{d}$ do not support three step sites along this line. In short, models $1 \mathrm{~b}-1 \mathrm{~d}$ cannot explain the STM topography of this step.

Model 1e is somewhat more promising. It consists of a bulk-like $2 \mathrm{~B}$ step plus a single dimer row. This structure supports three sites along the line $\mathbf{A}-\mathbf{A}^{\prime}$, but the location of site 'S2' seems to be incorrect. More specifically, in the STM side view, 'S2' and 'S3' do not appear to form the one-dimer-wide terrace predicted by model 1e.

Because none of the above models seems to fit our data, we developed an alternative (Fig. 1f). Our proposed model self-consistently explains the existence and location of all three step sites, assuming that each bright spot in the STM image corresponds to an atom on the surface. Note that this structure can be formed by adding two rows of atoms ('S2' and 'S3') to a pi-bonded 2B step (Fig. 1d). This alteration converts a 3fold site into a 5-fold site. Presumably, this 5-fold site must be occupied by an As atom, consistent with the fact that DR steps are not formed on clean Ge or Si surfaces.

Even if model $1 \mathrm{f}$ is correct, at least two additional details will need to be addressed. The first complication is that it is not known which atoms are Ge and which are As. One possibility is that all of the surface dimers and numbered atoms in our ball-andstick diagrams are As, whereas the other (bulk-like) atoms are Ge. In this case, each bright spot in our STM image would correspond to the lone electron pair of a surface As atom.

The second complication is that the presence of hydrogen on these surfaces could induce a myriad of structural permutations. Unfortunately, Auger electron spectroscopy is not sensitive to hydrogen, so we do not know if it is present on the surface. 


\section{RELATED STRUCTURES}

The 2B DR As/Ge step just discussed is merely the simplest structure out of an entire family of DR step structures. A thorough examination of Fig. 2 reveals many other DR structures. Although this image is particularly clear, it should be noted that we routinely observed the same DR structures for all three of the miscut angles/orientations we studied.

Most obvious are the type 'A' and 'B' DR steps. Despite the (110) miscut of this sample, all steps lie parallel and perpendicular to the dimer rows. In fact, this step direction is so stable that even small kinks are quite rare. In addition, the steps have bunched together to form multi-layer steps, which in turn bunch together to form sets

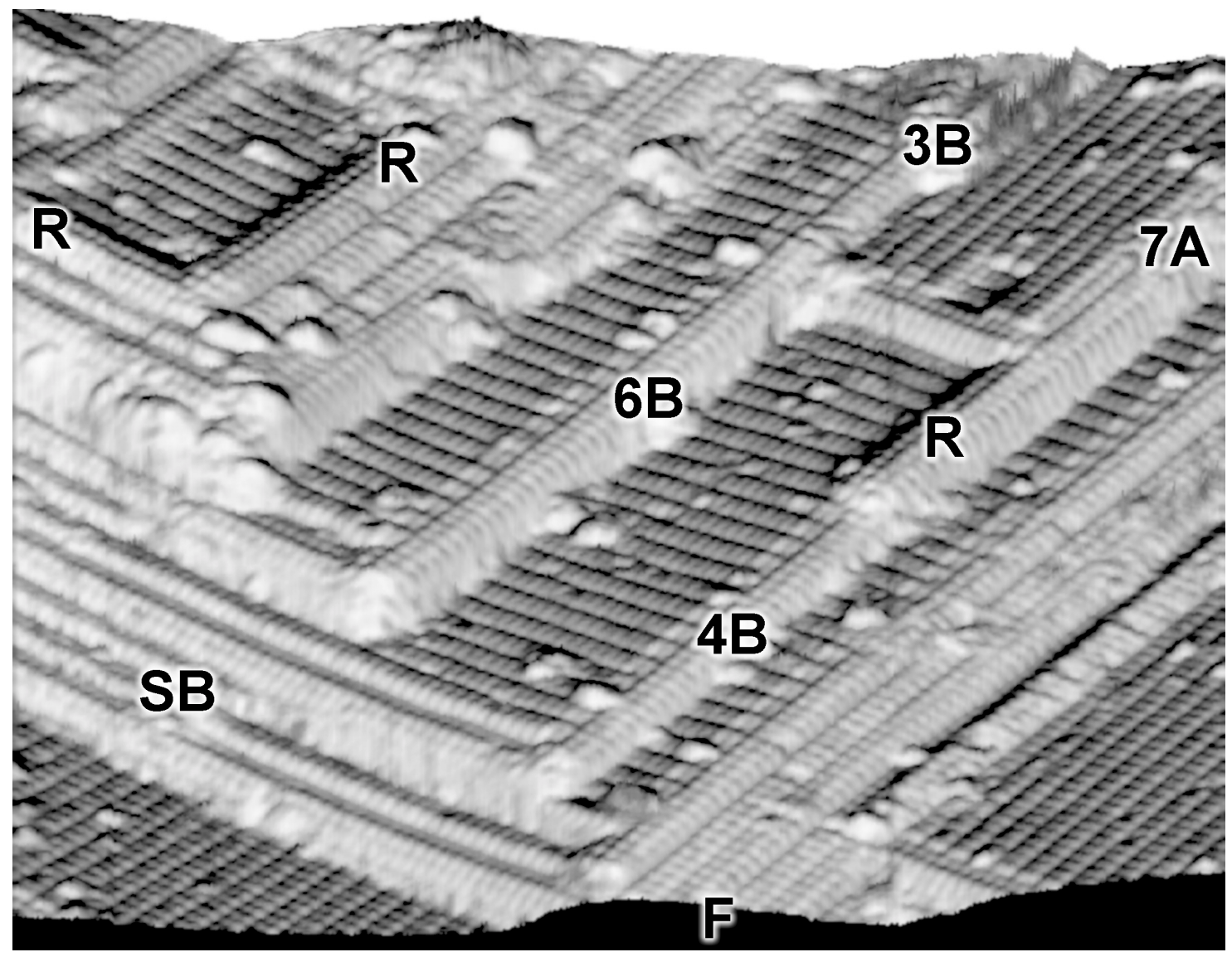

FIGURE 2. A $400 \AA$ x $400 \AA$ image of an As/Ge(100) $6^{\circ}$-(110) annealed in an MOCVD chamber under 1.2 torr $\mathrm{AsH}_{3}$ partial pressure for $20 \mathrm{~min}$ at $640^{\circ} \mathrm{C}$. This image has been artificially illuminated from behind the viewer. $\mathrm{V}_{\text {sample }}=-3.0 \mathrm{~V}$ and $\mathrm{I}_{\text {tun }}=0.3 \mathrm{nA}$.

Some representative DR steps are labeled with their height and type. 'SB' indicates a step-bunched region. ' $F$ ' indicates a region where the bunched steps have coalesced into a facet. Ridges are labeled with an 'R.' 
of multi-steps. Ultimately, these sets of multi-steps can coalesce into well-organized facets. Not surprisingly, the degree of facetting increases with miscut angle. Finally, $\mathrm{AsH}_{3}$ etching can remove the terrace behind a DR step, creating ridges. All of this is in sharp contrast to the step morphology of clean Ge, which typically consists of evenly spaced, heavily kinked, single-layer steps lying perpendicular to the miscut direction.

\section{CONCLUSIONS}

We have conducted an extensive STM study of arsenic-exposed vicinal Ge(100) surfaces and found that the resulting steps are reconstructed. We have observed two types of reconstructions, "single-row" reconstructions and "double-row" reconstructions. In this paper, we limited our discussion to double-row reconstructions resulting from arsine exposure, and proposed a structural model for a representative DR step.

On a vicinal substrate, steps obviously play an important role in all surface processes, including thin-film nucleation. We hope that a better understanding of As/Ge step structures will lead to a better understanding of GaAs/Ge nucleation.

\section{ACKNOWLEDGEMENTS}

This work would not have been possible without the help of J. Geisz, A. Kibbler, D. Friedman, S. Kurtz, C. Kramer, and K. Jones. In addition, we would like to thank S. Ringel, E. Fitzgerald, and R. Hicks for their helpful discussions. This work is supported by the Office of Energy Research, Basic Energy Sciences, U.S. Department of Energy.

\section{REFERENCES}

1. Bobb, L. C., Holloway, H., and Maxwell, K. H., J Appl Phys 37, 4687-4693 (1966).

2. Pelosi, C., Attolini, G., Bocchi, C., Franzosi, P., Frigeri, C., Berti, M., Drigo, A. V., and Romanato, F., J Electron Mater 24, 1723-1730 (1995).

3. Li, Y., Salviati, G., Bongers, M. M. G., Lazzarini, L., Nasi, L., and Giling, L. J., J Cryst Growth 163 , 195-202 (1996).

4. Chen, J. C., Ristow, M. L., Cubbage, J. I., and Werthen, J. G., J Electron Mater 21, 347-353 (1992).

5. Pukite, P. R., and Cohen, P. I., J Cryst Growth 81, 214-220 (1987).

6. Morar, J. F., Karlsson, U. O., Uhrberg, R. I. G., Kanski, J., Nilsson, P. O., and Qu, H., Appl Surf Sci 41/42, 312-316 (1989).

7. Alerhand, O. L., Wang, J., Joannopoulus, J. D., and Kaxiras, E., J Vac Sci Tech B9, 2423-2426 (1991).

8. Chadi, D. J., Phys Rev Lett 59, 1691-1694 (1987).

9. Aspnes, D. E., and Ihm, J., Phys Rev Lett 57, 3054-3057 (1986).

10. Itoh, H., Narui, S., Zhang, Z., and Ichonokawa, T., Surf Sci Lett 277, L70-L76 (1992). (The step we show is actually a variation of the steps presented in this reference.) 